\title{
Gd-EOB-DTPA-enhanced MRI radiomic features for predicting histological grade of hepatocellular carcinoma
}

\author{
Yingfan Mao ${ }^{1 \#}$, Jincheng Wang ${ }^{2 \#}$, Yong $\mathrm{Zhu}^{3}$, Jun Chen ${ }^{4}$, Liang Mao ${ }^{5}$, Weiwei Kong ${ }^{6}$, Yudong Qiu ${ }^{5}$, \\ Xiaoyan $\mathrm{Wu}^{1}$, Yue Guan ${ }^{7}$, Jian $\mathrm{He}^{1}$ \\ ${ }^{1}$ Department of Radiology, Nanjing Drum Tower Hospital, the Affiliated Hospital of Nanjing University Medical School, Nanjing, China; ${ }^{2}$ Department \\ of Hepatobiliary Surgery, Drum Tower Clinical Medical College, Nanjing Medical University, Nanjing, China; ${ }^{3}$ Department of Radiology, Jiangsu \\ Province Hospital of Traditional Chinese Medicine, the Affiliated Hospital of Nanjing University of Chinese Medicine, Nanjing, China; ${ }^{4}$ Department \\ of Pathology, Nanjing Drum Tower Hospital, the Affiliated Hospital of Nanjing University Medical School, Nanjing, China; ${ }^{5}$ Department of \\ Hepatopancreatobiliary Surgery, Nanjing Drum Tower Hospital, the Affiliated Hospital of Nanjing University Medical School, Nanjing, China; \\ ${ }^{6}$ Department of Oncology, Nanjing Drum Tower Hospital, the Affiliated Hospital of Nanjing University Medical School, Nanjing, China; ${ }^{7}$ School of \\ Biomedical Engineering, Shanghai Jiao Tong University, Shanghai, China \\ Contributions: (I) Conception and design: J He, X Wu; (II) Administrative support: Y Qiu, J He; (III) Provision of study materials or patients: J \\ Chen, L Mao, W Kong; (IV) Collection and assembly of data: Y Mao, Y Zhu; (V) Data analysis and interpretation: J Wang, Y Guan, Y Mao; (VI) \\ Manuscript writing: All authors; (VII) Final approval of manuscript: All authors. \\ "These authors contributed equally to this work. \\ Correspondence to: Jian He, MD; Xiaoyan Wu, BS. No. 321 Zhongshan Road, Nanjing 210008, China. Email: hjxueren@126.com; 1077wxy@163.com; \\ Yue Guan, PhD. No. 1954 Huashan Road, Shanghai 200030, China. Email: guan_yue@foxmail.com.
}

Background: Prediction models for the histological grade of hepatocellular carcinoma (HCC) remain unsatisfactory. The purpose of this study is to develop preoperative models to predict histological grade of HCC based on gadolinium-ethoxybenzyl diethylenetriamine pentaacetic acid (Gd-EOB-DTPA)-enhanced magnetic resonance imaging (MRI) radiomics. And to compare the performance between artificial neural network (ANN) and logistic regression model.

Methods: A total of 122 HCCs were randomly assigned to the training set $(n=85)$ and the test set $(n=37)$. There were 242 radiomic features extracted from volumetric of interest (VOI) of arterial and hepatobiliary phases images. The radiomic features and clinical parameters [gender, age, alpha-fetoprotein (AFP), carcinoembryonic antigen (CEA), carbohydrate antigen 19-9 (CA19-9), alanine aminotransferase (ALT), aspartate transaminase (AST)] were selected by permutation test and decision tree. ANN of arterial phase (ANN-AP), logistic regression model of arterial phase (LR-AP), ANN of hepatobiliary phase (ANN-HBP), logistic regression mode of hepatobiliary phase (LR-HBP), ANN of combined arterial and hepatobiliary phases $(\mathrm{ANN}-\mathrm{AP}+\mathrm{HBP})$, and logistic regression model of combined arterial and hepatobiliary phases (LR-AP + HBP) were built to predict HCC histological grade. Those prediction models were assessed and compared.

Results: ANN-AP and LR-AP were composed by AST and radiomic features based on arterial phase. ANN-HBP and LR-HBP were composed by AFP and radiomic features based on hepatobiliary phase. ANN-AP + HBP and LR-AP + HBP were composed by AST and radiomic features based on arterial and hepatobiliary phases. The prediction models could distinguish between high-grade tumors [EdmondsonSteiner (E-S) grade III and IV] and low-grade tumors (E-S grade I and II) in both training set and test set. In the test set, the AUCs of ANN-AP, LR-AP, ANN-HBP, LR-HBP, ANN-AP + HBP and LR-AP + HBP were $0.889,0.777,0.941,0.819,0.944$ and 0.792 respectively. The ANN-HBP was significantly superior to LR$\mathrm{HBP}(\mathrm{P}=0.001)$. And the ANN-AP + HBP was significantly superior to LR-AP + HBP $(\mathrm{P}=0.007)$.

Conclusions: Prediction models consisting of clinical parameters and Gd-EOB-DTPA-enhanced MRI radiomic features (based on arterial phase, hepatobiliary phase, and combined arterial and hepatobiliary phases) could distinguish between high-grade HCCs and low-grade HCCs. And the ANN was superior to logistic regression model in predicting histological grade of HCC. 
Keywords: Hepatocellular carcinoma (HCC); histological grade; magnetic resonance imaging (MRI); hepatobiliary phase; radiomics

Submitted Dec 02, 2019. Accepted for publication May 11, 2020.

doi: $10.21037 / \mathrm{hbsn}-19-870$

View this article at: http://dx.doi.org/10.21037/hbsn-19-870

\section{Introduction}

Hepatocellular carcinoma (HCC) is the fifth most common cancer in the world and the second leading cause of cancerrelated death (1). Surgical resection and liver transplantation are potential curative treatments for HCC patients (2), but postoperative recurrence is common. The recurrence rate of HCC after resection is up to $70 \%$ (3). Histological grade is one of the most important predictors of postoperative recurrence and prognosis in HCC patients (4-7). Compared with moderately differentiated and highly differentiated HCCs, poorly differentiated HCCs often indicate poor survival (8). Oishi et al. concluded that liver transplantation is not recommended for patients with poorly differentiated HCC $>3 \mathrm{~cm}$ (9). Okusaka et al. reported that patients with poorly differentiated HCC need more extensive treatment for a large safety margin and more frequent posttreatment follow-up (10). Biopsy, as the only preoperative method for obtaining tumor histopathological information, is invasive and has sampling errors. Thus, accurate assessment of HCC histological grade based on preoperative imaging is of paramount importance for treatment planning, prognosis, and postoperative management.

Radiomics is an emerging field of research that focus on high-throughput extraction of massive amounts of information and complex, hidden information based on statistics in medical images (11). Radiomics has been used for a variety of biological behavioral assessments (tumor recurrence, prognosis and histological grade) of various types of tumors (12-14). Computed tomography (CT) texture features, non-contrast-enhanced magnetic resonance imaging (MRI) radiomics signature, and Gd-DTPA contrast-enhanced MRI radiomics have been potentially applied to the prediction of preoperative HCC histological grade (15-17).

Gadolinium-ethoxybenzyl diethylenetriamine pentaacetic acid (Gd-EOB-DTPA) is a hepatocyte-specific contrast agent. Observational studies have confirmed that Gd-EOBDTPA-enhanced MRI plays an important role in predicting HCC histological grade (18-20). Recently, Kim et al. reported that radiomics on Gd-EOB-DTPA-enhanced MRI could predict early ( $\leq 2$ years) and late recurrence ( $>2$ years) after curative resection of single HCC patients (21). However, no one has attempted to predict histological grade of HCC with radiomic features on Gd-EOB-DTPAenhanced MRI.

Artificial neural network (ANN) is a commonly used machine learning method, consisting of a set of highly interconnected processing units (neurons), which are connected together by weighting (22). The network consists of input layer, output layer and one or more hidden layers. Hidden units enable neural networks to encode the nonlinear relationship between input and output (23). The interaction between many factors related to clinical decision is complex and nonlinear $(24,25)$. Traditional linear discriminant analysis is difficult to distinguish categories. $\mathrm{ANN}$ can fit the non-linear mapping of any relation with any accuracy for the multi-dimensional radiomics, and become a potential application tool to predict the clinical points $(26,27)$. In epidemiology and medicine, logistic regression is one of the most commonly used multivariate analysis for classification (28). ANN is found to be superior to logistic regression model in predict histopathological characteristics of HCC (29).

Thus, the purpose of this study was to develop preoperative models to predict histological grade of HCC based on Gd-EOB-DTPA-enhanced MRI radiomic features and clinical parameters. And to compare the performance between ANN and logistic regression model. We present the following article in accordance with the TRIPOD reporting checklist (available at https://hbsn.amegroups. com/article/view/10.21037/hbsn-19-870/rc).

\section{Methods}

\section{Patients}

The study was conducted in accordance with the Declaration of Helsinki (as revised in 2013). The study was approved by local institutional review board (No. 


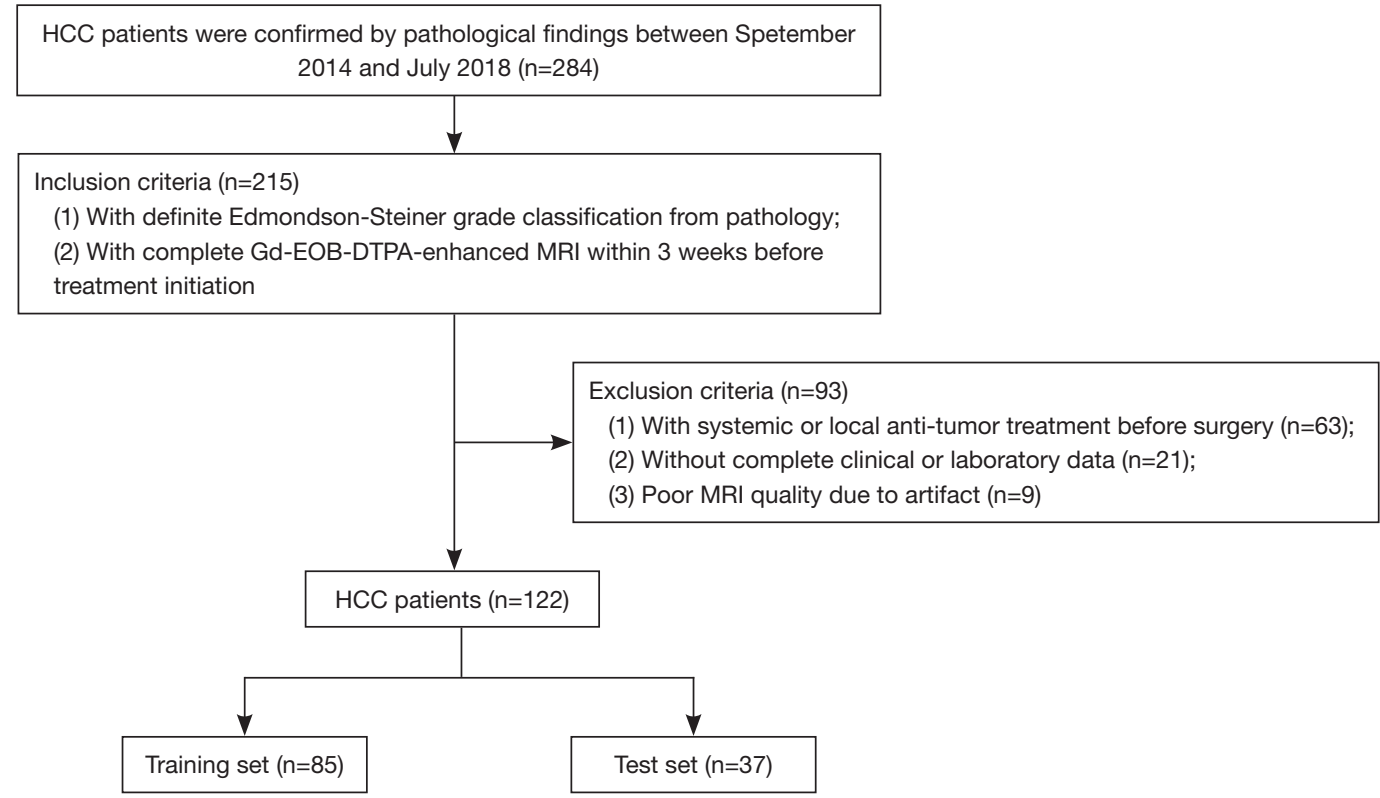

Figure 1 Flow-chart of inclusion and exclusion of this study. HCC, hepatocellular carcinoma; Gd-EOB-DTPA, gadolinium-ethoxybenzyl diethylenetriamine pentaacetic acid; MRI, magnetic resonance imaging.

2019AE01036), and informed consent from patients was waived due to its retrospective nature. From September 2014 to July 2018, 284 HCC patients were confirmed by pathological findings. The inclusion criteria were: (I) with definite Edmondson-Steiner (E-S) grade classification from postoperative pathology; (II) with complete Gd-EOBDTPA-enhanced MRI within 3 weeks before treatment initiation. The guidelines for receiving Gd-EOB-DTPAenhanced MRI in our institution referred to the updated Asian Pacific Association for the Study of the Liver (APASL) and Japan Society of Hepatology-Liver Cancer Study Group of Japan (JSH-LCSGJ) diagnostic algorithm (30,31). The exclusion criteria were: (I) with any systemic or local anti-tumor treatment such as radiofrequency or microwave ablation, transcatheter arterial chemoembolization (TACE), or molecular targeted therapy before surgery (n=63); (II) without complete clinical or laboratory data $(\mathrm{n}=21)$; (III) poor MRI quality due to artifact $(\mathrm{n}=9)$.

Finally, a total of 122 HCC patients were enrolled in our research cohort, 105 males and 17 females, with an average age of 58.2 \pm 10.1 years (range, 35-84 years). Patients were randomly assigned to the training set $(\mathrm{n}=85,70 \%)$ and the test set $(n=37,30 \%)$. The flowchart of inclusion and exclusion is shown in Figure 1. The clinicopathological information are summarized in Table 1. The flow-chart of the whole study is shown in Figure 2.
The clinical parameters, gender, age, alpha-fetoprotein (AFP), carcinoembryonic antigen (CEA), carbohydrate antigen 19-9 (CA19-9), alanine aminotransferase (ALT), and aspartate transaminase (AST), were obtained from electronic medical record system.

\section{MR examination}

All patients underwent routine Gd-EOB-DTPA-enhanced MR examination. Before the examination, each patient fasted for 6 hours and received breathing training. MR examination was performed using a whole body $3.0 \mathrm{~T}$ scanner (Ingenia 3.0 T, Philips Healthcare, Best, The Netherlands) with a 32 channels dStream Torso coil. The scan range was set from right side diaphragm to the level of the renal hilum. All patients received a rapid bolus of $0.025 \mathrm{mmol} / \mathrm{kg}$ body weight $(0.1 \mathrm{~mL} / \mathrm{kg})$ gadoxetic acid (Primovist; Bayer Schering Pharma AG, Berlin, Germany) at a rate of $1 \mathrm{~mL} / \mathrm{s}$, immediately followed by a $10 \mathrm{~mL}$ saline flush using an automatic power injector (Medrad Spectris Solaris EP MR Injector System; One Medrad Drive Indianola, PA, USA).

We obtained unenhanced and triple-arterial-phase (with a fixed 18-s scanning delay, each of these data sets lasted only $8 \mathrm{~s}$ ), portal-phase $(60 \mathrm{~s})$, late-phase $(3 \mathrm{~min})$, and hepatobiliary phase $(15 \mathrm{~min})$ images using a T1-weighted 
Table 1 The clinicopathological information of hepatocellular carcinoma patients

\begin{tabular}{|c|c|}
\hline Characteristics & Value \\
\hline \multicolumn{2}{|l|}{ Gender } \\
\hline Male & 105 \\
\hline Female & 17 \\
\hline Age (years) & $58.2 \pm 10.1$ \\
\hline \multicolumn{2}{|l|}{ Chronic viral hepatitis } \\
\hline Hepatitis B virus infection & 101 \\
\hline Hepatitis C virus infection & 9 \\
\hline Other & 12 \\
\hline \multicolumn{2}{|l|}{ Liver cirrhosis } \\
\hline With & 96 \\
\hline Without & 26 \\
\hline \multicolumn{2}{|l|}{ AFP } \\
\hline$>10 \mathrm{ng} / \mathrm{mL}$ & 78 \\
\hline$\leq 10 \mathrm{ng} / \mathrm{mL}$ & 44 \\
\hline \multicolumn{2}{|l|}{ CEA } \\
\hline$>5 \mathrm{ng} / \mathrm{mL}$ & 4 \\
\hline$\leq 5 \mathrm{ng} / \mathrm{mL}$ & 118 \\
\hline \multicolumn{2}{|l|}{ CA19-9 } \\
\hline$>39 \mathrm{U} / \mathrm{mL}$ & 17 \\
\hline$\leq 39 \mathrm{U} / \mathrm{mL}$ & 105 \\
\hline ALT & $38.6 \pm 37.5$ \\
\hline AST & $34.8 \pm 27.8$ \\
\hline \multicolumn{2}{|l|}{ TNM stage } \\
\hline I & 71 \\
\hline II & 41 \\
\hline III & 10 \\
\hline \multicolumn{2}{|l|}{ E-S grade } \\
\hline I & 14 \\
\hline II & 65 \\
\hline III & 41 \\
\hline IV & 2 \\
\hline
\end{tabular}

TNM stage is determined based on the 7th edition of the AJCC/UICC staging system. AFP, alpha-fetoprotein; CEA, carcinoembryonic antigen; CA19-9, carbohydrate antigen 19-9; ALT, alanine aminotransferase; AST, aspartate transaminase; E-S grade, Edmondson-Steiner grade classification; AJCC, American Joint Committee on Cancer; UICC, Union for International Cancer Control.
3D turbo-field-echo (TFE)sequence with a multi-echo Dixon fat-water separation technique. The triple-arterialphase and hepatobiliary phase MRI parameters included repetition time (TR)/echo time (TE), $3.7 \mathrm{~ms} / 1.32 \mathrm{~ms}$, $2.4 \mathrm{~ms}$; matrix size, $268 \times 236$; section thickness, $5 \mathrm{~mm}$; reconstruction section thickness, $2.5 \mathrm{~mm}$; field of view, 400×352; flip angle, $10^{\circ}$.

Axial T2 weighted images were obtained with respiratory-triggered turbo spin-echo sequence without fatsaturation (TR/TE, $582 \mathrm{~ms} / 70 \mathrm{~ms}$; matrix size, $252 \times 217$; section thickness, $5 \mathrm{~mm}$; field of view, $350 \times 400$; flip angle, $\left.90^{\circ}\right)$. The parameters for diffusion-weighted imaging $(\mathrm{b}=0$, $800 \mathrm{~s} / \mathrm{mm}^{2}$ ) were as follows: TR/TE, 2,944 ms/57 ms; matrix size, $120 \times 116$; section thickness, $6 \mathrm{~mm}$; field of view, $360 \times 360$; flip angle, $90^{\circ}$.

\section{MRI radiomics analysis}

Two radiologists (Y.M. and Y.Z., with 4 and 8 years' experience in abdominal radiology, respectively), who were blinded to clinicopathological information of patients, analyzed Gd-EOB-DTPA-enhanced MR images independently. The second phase of the triple-arterialphase (arterial phase) and hepatobiliary phase images at 2.5 -mm thickness (DICOM) of all patients were obtained from a picture archiving and communication system (PACS). We drew regions of interests (ROIs) of the whole tumor (including necrosis or cystic components) manually layer by layer on the arterial phase and hepatobiliary phase MR images separately with an in-house software (Image Analyzer 1.0, China), carefully avoiding the liver parenchyma and vessels around the tumor and the adjacent adipose tissue of abdominal cavity. Four tumors' boundary was slightly unclear on the arterial phase image. The completed ROI of tumor on hepatobiliary phase image was automatically copied to the arterial phase image. Then ROI on the arterial phase image was fine-tuned. Volumetric of interest (VOI) of tumor was obtained after reconstruction. A total of 121 radiomic features were extracted from each VOI, including histogram features $(\mathrm{n}=19)$, shape features $(\mathrm{n}=10)$, texture features $(\mathrm{n}=23 \times 4)$ from gray-level run-length matrix (GLRLM) and gray-level co-occurrence matrix (GLCM) (Table S1). To verify the robustness of the features, intraclass correlation coefficients (ICCs) were used to assess the interobserver agreement of the feature extractions. Features with an ICC lower than 0.75 were excluded from the final feature dataset. The average obtained by the two radiologists was used as the final result. 

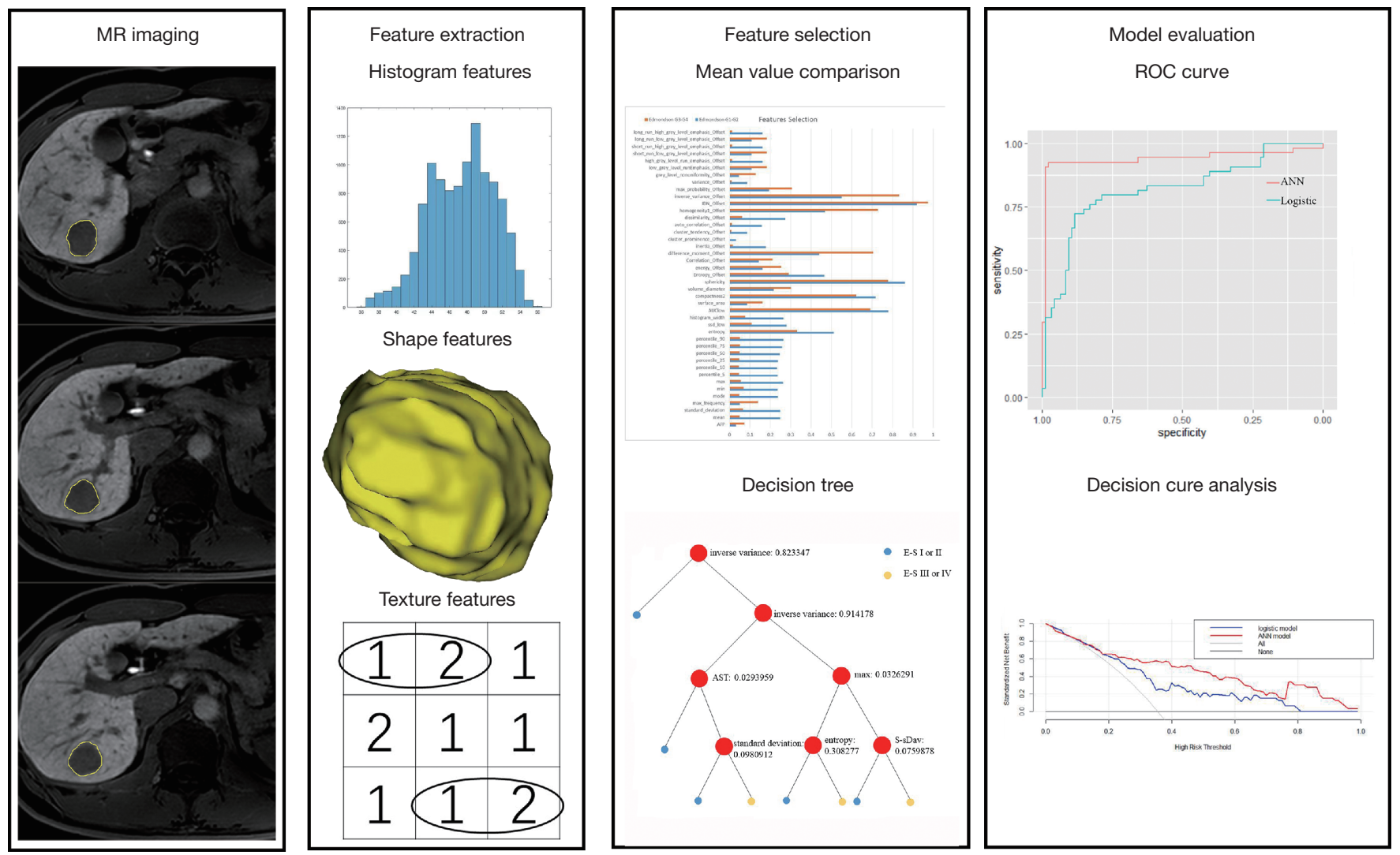

Figure 2 The flow-chart of the whole study. ROC, receiver operating characteristic.

\section{E-S grade}

A pathologist (J.C., with 15 years' experience in surgical pathology) reviewed hematoxylin-eosin staining slices microscopically to confirm histological grade of HCCs. According to the E-S grade classification, the histological grade was subclassified to E-S grade I to IV (32). E-S grade I and II corresponded to low-grade tumors, and E-S grade III and IV corresponded to high-grade tumors (33).

\section{Statistical analyses}

Shapiro-Wilk test and Levene test was used for normality and equality of variance respectively. ICC was firstly used to determine the reliability of each parameter between the two independent radiologists (ICC values greater than 0.75 indicate good reliability). The Gd-EOB DTPA-enhanced MRI radiomic features and preoperative clinical parameters were compared between low-grade tumors and high-grade tumors using permutation test. Decision tree was used for further parameter selection (34). Parameters selected by decision tree were used to build ANN and logistic regression model to predict histological grade of HCC. The probability of presence of the interest was obtained by the formula of logistic regression model: $p=1 /\left[1+e^{-\operatorname{logit}(\mathrm{p})}\right]$, and $\operatorname{logit}(\mathrm{p})=b_{0}+b_{1} x_{1}+b_{2} x_{2}+b_{3} x_{3}+b_{4} x_{4}+\ldots+b_{k} x_{k}$. ANN is a three-layer feedforward neural network with input nodes (clinical parameters or radiomic features), 23 neurons in the hidden layer, and one output neurons (high-grade HCC or low-grade HCC). The learning rule used here was back propagation of error, which adjusts the internal parameters of the network over the repeated training cycles to reduce the overall error (23). Area under a receiver operating characteristic (ROC) curve (AUC) was used to assess the performance of the prediction model. The best cutoff value for the output value of the model is obtained at the most approximate index. Delong's test (35) with a significant level of 0.05 was used to compare differences in AUCs of the prediction models. Decision curve analysis was used to assess the net benefits from different prediction models. Prediction models were 
built by Python 3.7 (Amsterdam, Netherlands). Other statistical analyses were conducted using $\mathrm{R}$ 3.5.1. A twotailed $\mathrm{P}$ value less than 0.05 was considered statistically significant.

\section{Results}

The baseline clinicopathological information of $122 \mathrm{HCC}$ patients is shown in Table 1. The 122 HCC specimens were separated into 79 low-grade tumors (14 E-S grade I tumors and $65 \mathrm{E}-\mathrm{S}$ grade II tumors) and 43 high-grade tumors (41 E-S grade III tumors and 2 E-S grade IV tumors).

\section{Interobserver reproducibility calculation}

AUC low (ICC value, 0.638), compactness 2 (ICC value, 0.513 ), sphericity (ICC value, 0.290), and cluster shade (ICC value, 0.655 ) were excluded from the arterial phase MRI radiomic dataset, and ICC values for the remaining 48 arterial phase MRI radiomic features between two radiologists ranged from 0.840 to 1.000 . Meanwhile, only sphericity (ICC value, 0.428 ) was excluded from the hepatobiliary phase MRI radiomic dataset, and ICC values for the remaining 51 hepatobiliary phase MRI radiomic features between two radiologists ranged from 0.757 to 1.000 (Table S2).

\section{Differences of clinical parameters and MRI radiomic features between low-grade tumors and high-grade tumors}

AFP and AST were significantly higher in patients with high-grade tumors than those with lower-grade tumors (505.77 \pm 685.94 vs. $230.10 \pm 824.16,46.62 \pm 44.94$ vs. $31.57 \pm 20.37, \mathrm{P}<0.001, \mathrm{P}=0.02$, respectively). MRI radiomic features based on arterial phase image $(n=39)$ and hepatobiliary phase image $(n=43)$ differed significantly between low-grade tumors and high-grade tumors (Table S3).

\section{Features selected by decision tree}

Decision tree selected AST and 5 radiomic features based on arterial phase MR image(standard deviation, maximum, entropy, s-sDav distribution width, inverse variance) for the LR-AP and ANN-AP (Figure S1A). Decision tree selected AFP and 8 radiomic features based on hepatobiliary phase MR image (max frequency, mode, minimum, the 10th percentile, s-sDlowest, AUC low, compactness2, inverse difference normalized) for the LR-HBP and ANN-HBP (Figure S1B).
At the same time, decision tree selected AST and 6 radiomic features based on arterial and hepatobiliary phases MR images (energy-AP, AUC low-HBP, mode-HBP, inverse variance$\mathrm{AP}$, inverse difference moment normalized-AP, maximum-AP) for LR-AP + HBP and ANN-AP + HBP (Figure S1C). The calculation formula or notes for the radiomic features selected by decision trees were showed in Supplement I.

\section{$A N N-A P$ and $L R-A P$}

ANN-AP has 6 input nodes (AST, standard deviation, maximum, entropy, s-sDav distribution width, inverse variance). The AUC, sensitivity and specificity of ANNAP were $0.945,0.980,0.962$ in the training set, and 0.889 , $0.913,0.882$ in the test set. Logit (p) of LR-AP $=0.285144 \times$ AST $-0.3999 \times$ standard deviation $+0.650041 \times$ maximum $+0.106613 \times$ entropy $-0.22434 \times \mathrm{s}-\mathrm{sDav}$ distribution width $+0.697344 \times$ inverse variance -0.14349 . The AUC, sensitivity and specificity of LR-AP were $0.805,0.627,0.962$ in the training set, and $0.777,0.652,0.882$ in the test set (Table 2) (Figure 3A).

\section{$A N N-H B P$ and LR-HBP}

ANN-HBP has 9 input nodes (AFP, max frequency, mode, minimum, the 10th percentile, s-sDlowest, AUC low, compactness 2 , inverse difference normalized). The AUC, sensitivity and specificity of ANN-HBP were $0.975,1.000$, 0.970 in the training set, and $0.941,0.979,0.926$ in the test set. Logit (p) of LR-HBP $=-0.19038 \times$ AFP $+1.07289 \times$ max frequency $+9.477355 \times$ mode $+1.265287 \times$ minimum $-13.3298 \times$ the 10 th percentile $+1.911985 \times$ s-sDlowest $-0.02488 \times$ AUC low $-0.49382 \times$ compactness $2-0.1581$ $\times$ inverse difference normalized +0.724399 . The AUC, sensitivity and specificity of LR-HBP were $0.820,0.783$, 0.788 in the training set, and $0.819,0.883,0.722$ in the test set (Table 2) (Figure 3B).

\section{$A N N-A P+H B P$ and $L R-A P+H B P$}

ANN-AP + HBP has 7 input nodes (AST, energy-AP, AUC low-HBP, mode-HBP, inverse variance-AP, inverse difference moment normalized-AP, maximum-AP), the AUC, sensitivity and specificity of ANN-AP + HBP were $0.953,1.000,0.792$ in the training set, and $0.944,0.840$, 0.998 in the test set. Logit $(\mathrm{p})$ of LR-AP $+\mathrm{HBP}=0.601374$ $\times$ AST $-0.147834 \times$ mode $(\mathrm{HBP})-0.218004 \times$ AUC low $(\mathrm{HBP})-0.149234 \times$ maximum $(\mathrm{AP})-0.596663 \times$ energy 
Table 2 Performance of ANN model and LR model in differentiating different histological grades

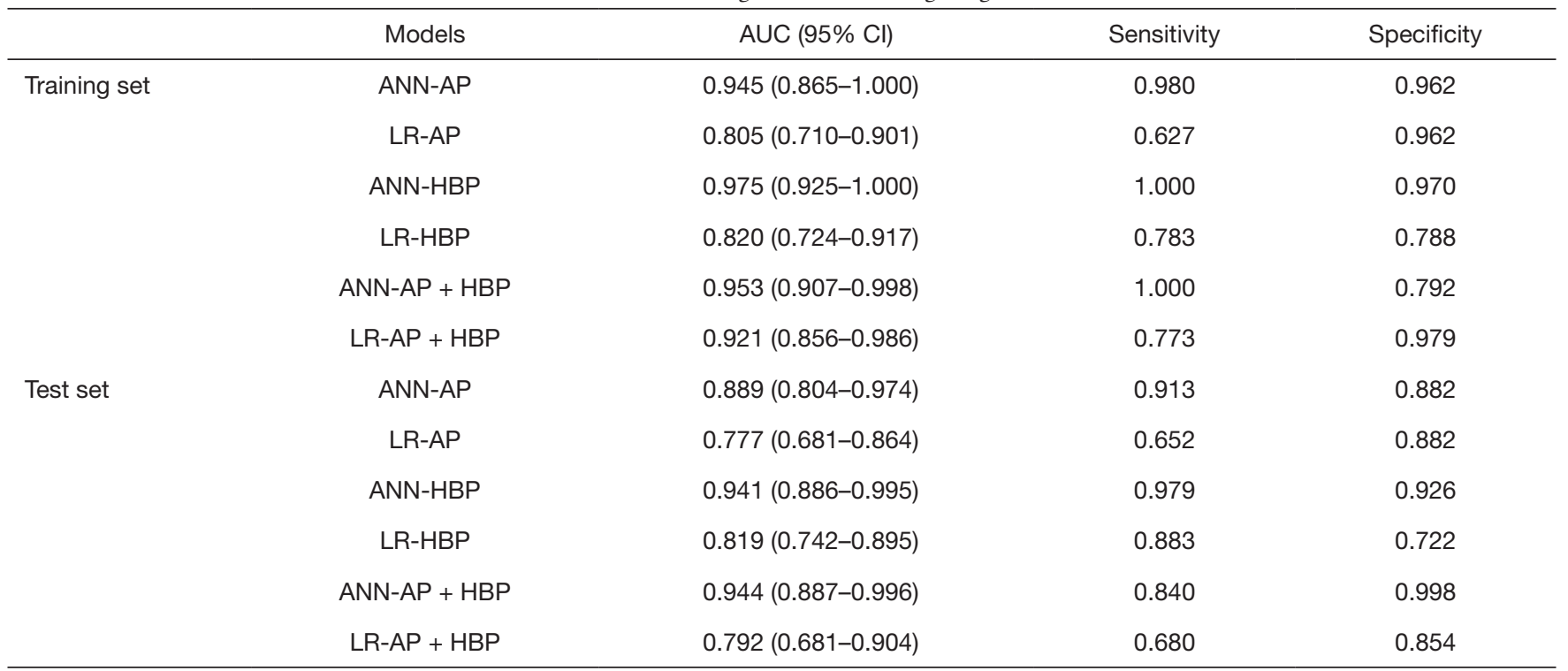

ANN, artificial neural network; LR, logistic regression; AP, arterial phase; HBP, hepatobiliary phase; AUC, area under the receiver operating characteristic curve; $\mathrm{Cl}$, confidence interval.

$(\mathrm{AP})+2.033877 \times$ inverse difference moment normalized $(\mathrm{AP})-1.153323 \times$ inverse variance $(\mathrm{AP})+0.272895$. The AUC, sensitivity and specificity of LR-AP + HBP were $0.921,0.773,0.979$ in the training set, and $0.792,0.680$, 0.854 in the test set (Table 2) (Figure 3C).

\section{Comparison for different prediction models in the test set}

As shown in Table 3, the difference between ANN-HBP and the LR-HBP was statistically significant $(\mathrm{P}=0.001)$. And there was significant difference between ANN-AP + $\mathrm{HBP}$ and the LR-AP + HBP $(\mathrm{P}=0.007)$. As shown in Figure $4 A$, ANN-AP provided a better net benefit to differentiate HCC grades than LR-AP for threshold probabilities of $50-66 \%$ in the test set. As shown in Figure 4B, ANN-HBP provided a better net benefit to differentiate HCC grades than LR-HBP for threshold probabilities of more than $20 \%$ in the test set. As shown in Figure 4C, ANN-AP + HBP provided a better net benefit to differentiate HCC grades than LR-AP + HBP for threshold probabilities of more than $10 \%$ in the test set.

\section{Discussion}

We compared the differences of the clinical parameters and Gd-EOB-DTPA enhanced MRI radiomic features (based on arterial phase and hepatobiliary phase) between low- grade HCCs and high-grade HCCs. ANN and logistic regression models composed of MRI radiomic features and clinical parameters were established for predicting histological grades of HCC preoperatively.

There were significant differences in MRI radiomic features (based on arterial phase and hepatobiliary phase) between low-grade and high-grade HCCs. Histogram reflects heterogeneity or distribution of voxel values and texture reflects the relationship of voxel measures with neighboring voxels. Many studies have shown that the relative signal intensity of tumors $(18,20)$ and subjective imaging features (36) on the arterial phase and hepatobiliary phase MR images different significantly between highgrade and low grade HCCs. Taking voxels as a unit, we analyzed the distribution of signals on the arterial phase and hepatobiliary phase images. We proved that a large number of hidden radiomic features had significant differences between different grades of HCC, which were expected to become a new tool for predicting HCC histological grade.

We found significant differences in entropy and entropy $(\mathrm{H})$ on arterial phase and hepatobiliary phase MR images between low-grade and high-grade HCCs. Although no definite relationship between entropy (based on MRI texture analysis) and histological grade of HCC has been reported, some studies have reported that entropy in the hepatobiliary phase was significantly associated with early recurrence and prognosis in HCC patients $(37,38)$. In addition, entropy 

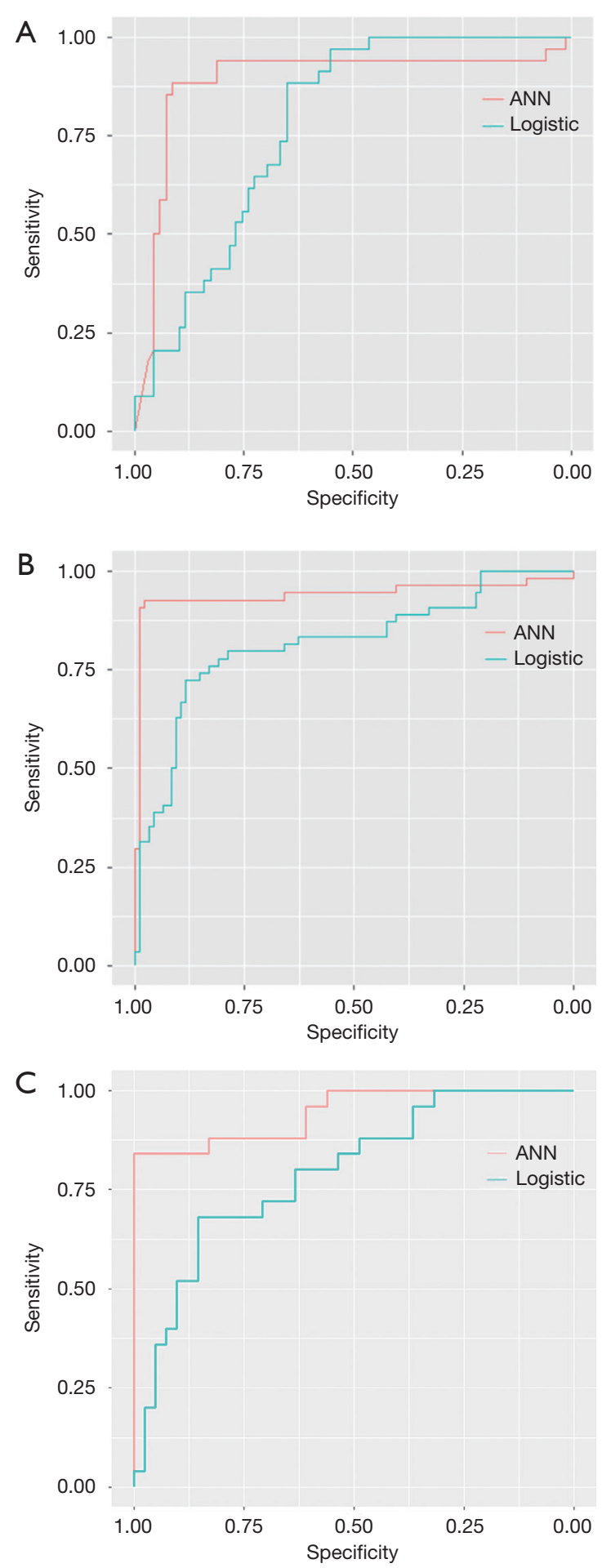

Figure 3 ROC curve for differentiating different histological grades in test set: comparison between ANN-AP and LR-AP (A), comparison between ANN-HBP and LR-HBP (B), and comparison between ANN-AP + HBP and LR-AP + HBP (C). ROC, receiver operating characteristic; ANN, artificial neural network; AP, arterial phase; LR, logistic regression; HBP, hepatobiliary phase.
Table 3 Delong test for the prediction models in the test set

\begin{tabular}{lc}
\hline Models & P value \\
\hline ANN-AP vs. LR-AP & 0.093 \\
ANN-HBP vs. LR-HBP & $0.001^{\star}$ \\
ANN-AP + HBP vs. LR-AP + HBP & $0.007^{\star}$ \\
ANN-AP vs. ANN-HBP & 0.318 \\
ANN-AP vs. ANN-AP + HBP & 0.306 \\
ANN-HBP vs. ANN-AP + HBP & 0.974 \\
LR-AP vs. LR-HBP & 0.487 \\
LR-AP vs. LR-AP + HBP & 0.633 \\
LR-HBP vs. LR-AP + HBP & 0.524
\end{tabular}

*, $\mathrm{P}<0.05$. ANN, artificial neural network; LR, logistic regression; AP, arterial phase; HBP, hepatobiliary phase.

based on MRI texture analysis was confirmed to be effective for grading renal cell carcinoma, pancreatic neuroendocrine tumor and gliomas (39-41). In this study, we also found that some histogram features on arterial phase and hepatobiliary phase MR images (mean, minimum, maximum, the nth percentile, etc.) of low-grade tumors were significantly higher than those of high-grade tumors. Zhou et al. had similar findings as us, they demonstrated that the mean intensity value in the arterial phase of low-grade HCCs was significantly higher than that of high-grade HCCs, which may be owing to more necrotic components in the highgrade HCCs (17). Chang et al. illustrated that low-grade HCCs had significantly higher relative intensity ratio (signal intensities of the HCC/signal intensities of the adjacent liver parenchyma) in the arterial phase and hepatobiliary phase than high-grade HCCs (42), which also supported our findings. Oh et al. found that the mean of positive pixels of HCCs based on CT texture analysis in the arterial phase was positively correlated with histological grade of tumors (15), which was not in conflict with our findings, because the mean on the arterial phase images in our study was the average pixel value including the necrotic components.

In our study, prediction models based on Gd-EOBDTPA-enhanced MRI radiomic features could distinguish between high-grade HCCs and low-grade HCCs, with AUCs of $0.805-0.975$ in the training set and AUCs of 0.777 to 0.944 in the test set. Zhou et al. declared that the mean value and grey-level run-length non-uniformity (GLN) based on Gd-DTPA-enhanced MRI texture analysis were associated with the pathological grade of HCC (with 
A

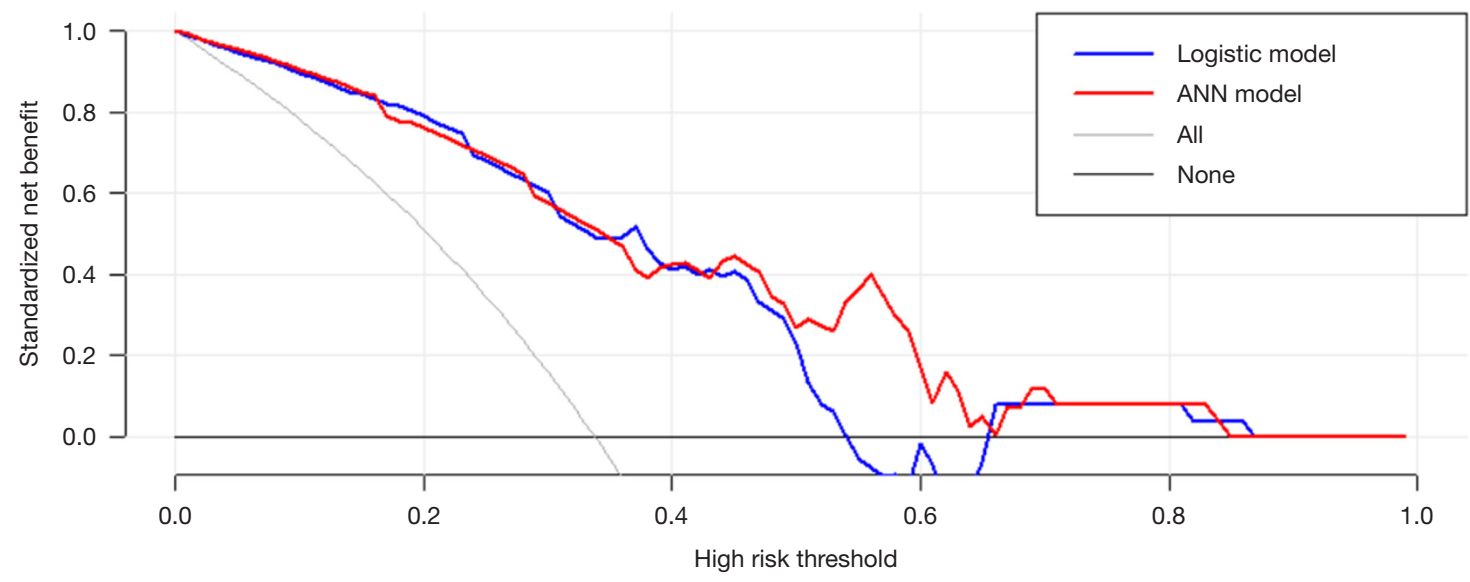

B

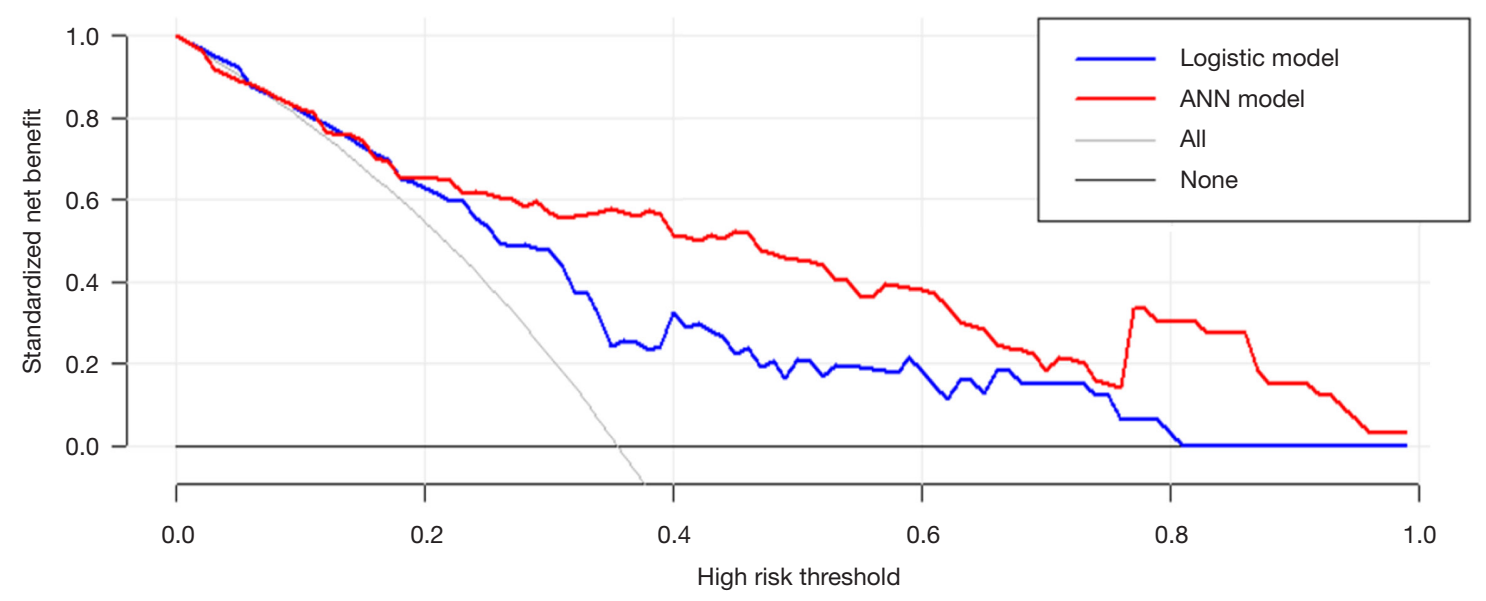

C

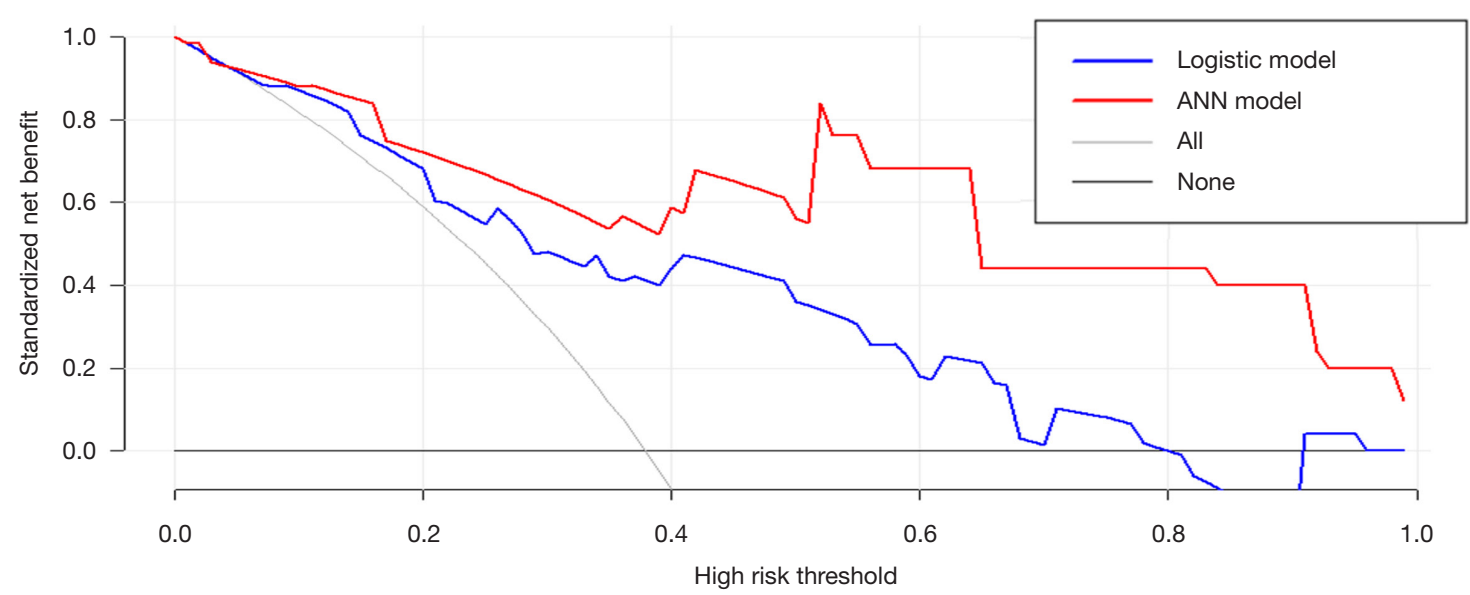

Figure 4 Decision curve analysis for the prediction model evaluation in the test set: comparison between ANN-AP and LR-AP (A), comparison between ANN-HBP and LR-HBP (B), and comparison between ANN-AP + HBP and LR-AP + HBP (C). ANN, artificial neural network; AP, arterial phase; LR, logistic regression; HBP, hepatobiliary phase. 
AUCs of $0.827-0.918$ ) (17), but they had a very limited number of cases, with only 46 HCC patients, and without further testing. Wu et al. established the combined models composed of clinical factors and radiomics signatures (based on T1WI images, T2WI images and combined T1WI and T2WI images) to distinguish between low grade tumors (E-S grades I, I-II and II) and high grade tumors (E-S grades II-III, III, III-IV and IV), with AUCs from 0.742 to 0.800 for the test dataset (16), which was inferior to us. This may be because Gd-EOB-DTPA-enhanced MRI radiomic features can provide more information about the heterogeneity of tumors. We found no significant difference between ANN-AP and ANN-HBP in distinguishing HCC grades. And there was no significant difference between LR$\mathrm{AP}$ and LR-HBP in distinguishing HCC grades. However, Chang et al. believed that the relative intensity ratio (signal intensities of the HCC/signal intensities of the adjacent liver parenchyma) in the arterial phase performed better than that in the hepatobiliary phase in grading HCCs (AUC, 0.95 vs. 0.70) (42). They believed that the background of cirrhosis weakened the ability of signal intensities in the hepatobiliary phase of HCCs to differentiate tumor grade. Nevertheless, a large proportion of patients with cirrhosis were also included in our cohort $(77 / 122,63.11 \%)$. Of course, research methods are fundamentally different between us, and the MRI radiomic features based on hepatobiliary phase may have captured more information. We found that ANN-AP performed better than LR-AP, ANN-AP + HBP performed better than LR-AP + HBP in grading HCCs. ANN is superior to the conventional statistical linear approach, which is increasingly being used in clinical research (43). Cucchetti et al. also found that ANN model performed better than logistic regression model in predicting tumor grade of HCC (AUC, $0.94 v s$. 0.85), which confirmed our results (29).

There were some limitations in our research. Firstly, it was a single-center retrospective study in which selective bias was inevitable. Although we have done internal validation, further external validation is needed to confirm the reliability of the model. Secondly, the number of E-S grade I and E-S grade IV was limited, but we concentrated on the differentiation between low-grade tumors and highgrade tumors, rather than between four E-S grades. Thirdly, different MR machines and scanning parameters may affect the extraction of radiomic features, which may limit the application of the model to some extent. Finally, our models only contained some clinical parameters and MRI radiomic features (based on arterial phase and hepatobiliary phase).
In the future, we will try to analyze macroscopic imaging features of Gd-EOB-DTPA-enhanced MRI and radiomic features of more sequences of MRI.

In conclusion, our study found that combined models consisting of clinical parameters and Gd-EOB-DTPAenhanced MRI radiomic features (based on arterial phase, hepatobiliary phase, and combined arterial and hepatobiliary phases) could distinguish between high-grade HCCs and low-grade HCCs. And the ANN was superior to logistic regression model in distinguishing $\mathrm{HCC}$ histological grades.

\section{Acknowledgments}

The authors thank the hepatobiliary pancreatic tumor multidisciplinary team in Nanjing Drum Tower Hospital. Funding: This study has received funding by Outstanding Youth supported by Medical Science and Technology Development Foundation Nanjing (JQX16022), Jiangsu Province Key Medical Young Talents, "13th FiveYear" Health Promotion Project of Jiangsu Province (QNRC2016041).

\section{Footnote}

Reporting Checklist: The authors have completed the TRIPOD reporting checklist. Available at https://hbsn. amegroups.com/article/view/10.21037/hbsn-19-870/rc

Data Sharing Statement: Available at https://hbsn. amegroups.com/article/view/10.21037/hbsn-19-870/dss

Conflicts of Interest: All authors have completed the ICMJE uniform disclosure form (available at https://hbsn. amegroups.com/article/view/10.21037/hbsn-19-870/coif). The authors have no conflicts of interest to declare.

Ethical Statement: The authors are accountable for all aspects of the work in ensuring that questions related to the accuracy or integrity of any part of the work are appropriately investigated and resolved. The study was conducted in accordance with the Declaration of Helsinki (as revised in 2013). The study was approved by local institutional review board (No. 2019AE01036), and informed consent from patients was waived due to its retrospective nature.

Open Access Statement: This is an Open Access article 
distributed in accordance with the Creative Commons Attribution-NonCommercial-NoDerivs 4.0 International License (CC BY-NC-ND 4.0), which permits the noncommercial replication and distribution of the article with the strict proviso that no changes or edits are made and the original work is properly cited (including links to both the formal publication through the relevant DOI and the license). See: https://creativecommons.org/licenses/by-nc-nd/4.0/.

\section{References}

1. Torre LA, Bray F, Siegel RL, et al. Global cancer statistics, 2012. CA Cancer J Clin 2015;65:87-108.

2. Kanwal F, Befeler A, Chari RS, et al. Potentially curative treatment in patients with hepatocellular cancer--results from the liver cancer research network. Aliment Pharmacol Ther 2012;36:257-65.

3. Tabrizian P, Jibara G, Shrager B, et al. Recurrence of hepatocellular cancer after resection: patterns, treatments, and prognosis. Ann Surg 2015;261:947-55.

4. Lauwers GY, Terris B, Balis UJ, et al. Prognostic histologic indicators of curatively resected hepatocellular carcinomas: a multi-institutional analysis of 425 patients with definition of a histologic prognostic index. Am J Surg Pathol 2002;26:25-34.

5. Jonas S, Bechstein WO, Steinmuller T, et al. Vascular invasion and histopathologic grading determine outcome after liver transplantation for hepatocellular carcinoma in cirrhosis. Hepatology 2001;33:1080-6.

6. Zhou L, Rui JA, Wang SB, et al. Factors predictive for long-term survival of male patients with hepatocellular carcinoma after curative resection. J Surg Oncol 2007;95:298-303

7. Zhou L, Rui JA, Ye DX, et al. Edmondson-Steiner grading increases the predictive efficiency of TNM staging for long-term survival of patients with hepatocellular carcinoma after curative resection. World J Surg 2008;32:1748-56.

8. Court CM, Harlander-Locke MP, Markovic D, et al. Determination of hepatocellular carcinoma grade by needle biopsy is unreliable for liver transplant candidate selection. Liver Transpl 2017;23:1123-32.

9. Oishi K, Itamoto T, Amano H, et al. Clinicopathologic features of poorly differentiated hepatocellular carcinoma. J Surg Oncol 2007;95:311-6.

10. Okusaka T, Okada S, Ueno H, et al. Satellite lesions in patients with small hepatocellular carcinoma with reference to clinicopathologic features. Cancer 2002;95:1931-7.
11. Lambin P, Rios-Velazquez E, Leijenaar R, et al. Radiomics: extracting more information from medical images using advanced feature analysis. Eur J Cancer 2012;48:441-6.

12. Jiang $\mathrm{Y}$, Chen $\mathrm{C}$, Xie J, et al. Radiomics signature of computed tomography imaging for prediction of survival and chemotherapeutic benefits in gastric cancer. EBioMedicine 2018;36:171-82.

13. Thawani R, McLane M, Beig N, et al. Radiomics and radiogenomics in lung cancer: A review for the clinician. Lung Cancer 2018;115:34-41.

14. Huang X, Cheng Z, Huang Y, et al. CT-based Radiomics Signature to Discriminate High-grade From Low-grade Colorectal Adenocarcinoma. Acad Radiol 2018;25:1285-97.

15. Oh J, Lee JM, Park J, et al. Hepatocellular Carcinoma: Texture Analysis of Preoperative Computed Tomography Images Can Provide Markers of Tumor Grade and Disease-Free Survival. Korean J Radiol 2019;20:569-79.

16. Wu M, Tan H, Gao F, et al. Predicting the grade of hepatocellular carcinoma based on non-contrast-enhanced MRI radiomics signature. Eur Radiol 2019;29:2802-11.

17. Zhou W, Zhang L, Wang K, et al. Malignancy characterization of hepatocellular carcinomas based on texture analysis of contrast-enhanced MR images. J Magn Reson Imaging 2017;45:1476-84.

18. Kim HY, Choi JY, Kim CW, et al. Gadolinium ethoxybenzyl diethylenetriamine pentaacetic acidenhanced magnetic resonance imaging predicts the histological grade of hepatocellular carcinoma only in patients with Child-Pugh class A cirrhosis. Liver Transpl 2012;18:850-7.

19. An C, Park MS, Jeon HM, et al. Prediction of the histopathological grade of hepatocellular carcinoma using qualitative diffusion-weighted, dynamic, and hepatobiliary phase MRI. Eur Radiol 2012;22:1701-8.

20. Kogita S, Imai Y, Okada M, et al. Gd-EOB-DTPAenhanced magnetic resonance images of hepatocellular carcinoma: correlation with histological grading and portal blood flow. Eur Radiol 2010;20:2405-13.

21. Kim S, Shin J, Kim DY, et al. Radiomics on Gadoxetic Acid-Enhanced Magnetic Resonance Imaging for Prediction of Postoperative Early and Late Recurrence of Single Hepatocellular Carcinoma. Clin Cancer Res 2019;25:3847-55.

22. Cross SS, Harrison RF, Kennedy RL. Introduction to neural networks. Lancet 1995;346:1075-9.

23. Kemp RA, MacAulay C, Palcic B. Opening the black box: the relationship between neural networks and linear discriminant functions. Anal Cell Pathol 1997;14:19-30. 
24. Pawlik TM, Delman KA, Vauthey JN, et al. Tumor size predicts vascular invasion and histologic grade: Implications for selection of surgical treatment for hepatocellular carcinoma. Liver Transpl 2005;11:1086-92.

25. Pawlik TM, Abdalla EK, Vauthey JN. Liver transplantation for hepatocellular carcinoma: need for a new patient selection strategy. Ann Surg 2004;240:923-4, 924-5.

26. Penny $W$, Frost D. Neural networks in clinical medicine. Med Decis Making 1996;16:386-98.

27. Cucchetti A, Vivarelli M, Heaton ND, et al. Artificial neural network is superior to MELD in predicting mortality of patients with end-stage liver disease. Gut 2007;56:253-8.

28. Kasza J, Wolfe R. Interpretation of commonly used statistical regression models. Respirology 2014;19:14-21.

29. Cucchetti A, Piscaglia F, Grigioni AD, et al. Preoperative prediction of hepatocellular carcinoma tumour grade and micro-vascular invasion by means of artificial neural network: a pilot study. J Hepatol 2010;52:880-8.

30. Kudo M, Matsui O, Izumi N, et al. JSH ConsensusBased Clinical Practice Guidelines for the Management of Hepatocellular Carcinoma: 2014 Update by the Liver Cancer Study Group of Japan. Liver Cancer 2014;3:458-68.

31. Omata M, Cheng AL, Kokudo N, et al. Asia-Pacific clinical practice guidelines on the management of hepatocellular carcinoma: a 2017 update. Hepatol Int 2017;11:317-70.

32. Amin MB. AJCC cancer staging manual. Eight edition. Amin MB. Edge SB. 2017.

33. Edmondson HA, Steiner PE. Primary carcinoma of the liver: a study of 100 cases among 48,900 necropsies. Cancer 1954;7:462-503.

34. Torlay L, Perrone-Bertolotti M, Thomas E, et al. Machine learning-XGBoost analysis of language networks to classify patients with epilepsy. Brain Inform 2017;4:159-69.

35. DeLong ER, DeLong DM, Clarke-Pearson DL.

Cite this article as: Mao Y, Wang J, Zhu Y, Chen J, Mao L, Kong W, Qiu Y, Wu X, Guan Y, He J. Gd-EOB-DTPAenhanced MRI radiomic features for predicting histological grade of hepatocellular carcinoma. HepatoBiliary Surg Nutr 2022;11(1):13-24. doi: 10.21037/hbsn-19-870
Comparing the areas under two or more correlated receiver operating characteristic curves: a nonparametric approach. Biometrics 1988;44:837-45.

36. Chen CY, Chen J, Xia CC, et al. T1 mapping combined with Gd-EOB-DTPA-enhanced magnetic resonance imaging in predicting the pathologic grading of hepatocellular carcinoma. J Biol Regul Homeost Agents 2017;31:1029-36.

37. Ahn SJ, Kim JH, Park SJ, et al. Hepatocellular carcinoma: preoperative gadoxetic acid-enhanced MR imaging can predict early recurrence after curative resection using image features and texture analysis. Abdom Radiol (NY) 2019;44:539-48.

38. Rosenkrantz AB, Pinnamaneni N, Kierans AS, et al. Hypovascular hepatic nodules at gadoxetic acid-enhanced MRI: whole-lesion hepatobiliary phase histogram metrics for prediction of progression to arterial-enhancing hepatocellular carcinoma. Abdom Radiol (NY) 2016;41:63-70.

39. Goyal A, Razik A, Kandasamy D, et al. Role of MR texture analysis in histological subtyping and grading of renal cell carcinoma: a preliminary study. Abdom Radiol (NY) 2019;44:3336-49.

40. Guo CG, Ren S, Chen X, et al. Pancreatic neuroendocrine tumor: prediction of the tumor grade using magnetic resonance imaging findings and texture analysis with 3-T magnetic resonance. Cancer Manag Res 2019;11:1933-44.

41. Ditmer A, Zhang B, Shujaat T, et al. Diagnostic accuracy of MRI texture analysis for grading gliomas. J Neurooncol 2018;140:583-9.

42. Chang WC, Chen RC, Chou CT, et al. Histological grade of hepatocellular carcinoma correlates with arterial enhancement on gadoxetic acid-enhanced and diffusionweighted MR images. Abdom Imaging 2014;39:1202-12.

43. Baxt WG. Application of artificial neural networks to clinical medicine. Lancet 1995;346:1135-8. 


\section{Supplementary}

Table S1 Gd-EOB-DTPA-enhanced MRI radiomic features extracted from the volumetric of interest of hepatocellular carcinoma

\begin{tabular}{ll}
\hline Category & Features \\
\hline Histogram features $(\mathrm{n}=19)$ & $\begin{array}{l}\text { Mean, standard deviation, max frequency, mode, minimum, maximum, the 5th percentile, the 10th } \\
\text { percentile, the 25th percentile, the 50th percentile, the 75th percentile, the 90th percentile, skewness, } \\
\text { kurtosis, entropy, AUC low, s-sDlowest, s-sDav distribution width, histogram width }\end{array}$ \\
Shape features $(\mathrm{n}=10)$ & $\begin{array}{l}\text { Area, volume, surface area, compactness1, compactness2, volume diameter, spherical disproportion, } \\
\text { sphericity, surface volume ratio, elongation }\end{array}$ \\
Texture features $(\mathrm{n}=23 \times 4)$ & $\begin{array}{l}\text { Entropy }(\mathrm{H}) \text {, energy, correlation, inverse difference moment normalized, cluster shade, cluster prominence, } \\
\text { cluster tendency, auto correlation, dissimilarity, homogeneity1, homogeneity2, inverse difference } \\
\text { normalized, inverse variance, max probability, variance, grey level nonuniformity, run length nonuniformity, } \\
\text { low grey level run emphasis, high grey level run emphasis, short run low grey level emphasis, short run high } \\
\text { grey level emphasis, long run low grey level emphasis, long run high grey level emphasis }\end{array}$ \\
\hline
\end{tabular}

Texture features $(23 \times 4)$ were extracted from four GLRLMs and four GLCMs, when the direction angle $\theta$ of matrix was set to $0^{\circ}, 45^{\circ}, 90^{\circ}$ and $135^{\circ}$ respectively. Gd-EOB-DTPA, gadolinium-ethoxybenzyl diethylenetriamine pentaacetic acid; MRI, magnetic resonance imaging. 
Table S2 Intraclass correlation coefficients (ICC) for Gd-EOB-DTPA-enhanced MRI radiomic features based on the arterial phase and hepatobiliary phase between two radiologists

\begin{tabular}{|c|c|c|}
\hline Radiomic features & ICC (arterial phase) & ICC (hepatobiliary phase) \\
\hline Mean & 1.000 & 1.000 \\
\hline Standard deviation & 0.998 & 0.959 \\
\hline Max frequency & 1.000 & 1.000 \\
\hline Mode & 0.999 & 1.000 \\
\hline Minimum & 0.961 & 0.990 \\
\hline Maximum & 0.999 & 0.997 \\
\hline The 5th percentile & 0.999 & 0.999 \\
\hline The 10th percentile & 1.000 & 1.000 \\
\hline The 25th percentile & 1.000 & 1.000 \\
\hline The 50th percentile & 1.000 & 1.000 \\
\hline The 75th percentile & 1.000 & 0.999 \\
\hline The 90th percentile & 1.000 & 0.998 \\
\hline Skewness & 0.911 & 0.970 \\
\hline Kurtosis & 0.840 & 0.964 \\
\hline Entropy & 1.000 & 0.987 \\
\hline AUC low & $0.638^{*}$ & 0.944 \\
\hline S-sDlowest & 0.993 & 0.999 \\
\hline S-sDav distribution width & 0.894 & 0.997 \\
\hline Histogram width & 0.980 & 0.962 \\
\hline Area & 1.000 & 1.000 \\
\hline Volume & 1.000 & 1.000 \\
\hline Surface area & 1.000 & 1.000 \\
\hline compactness1 & 0.999 & 0.995 \\
\hline compactness2 & $0.513^{*}$ & 0.757 \\
\hline Volume diameter & 1.000 & 1.000 \\
\hline Spherical disproportion & 0.928 & 0.922 \\
\hline Sphericity & $0.290^{*}$ & $0.428^{*}$ \\
\hline Surface volume ratio & 0.921 & 0.986 \\
\hline Elongation & 1.000 & 1.000 \\
\hline Entropy $(\mathrm{H})$ & 0.999 & 0.995 \\
\hline Energy & 0.999 & 0.998 \\
\hline Correlation & 0.998 & 0.994 \\
\hline Inverse difference moment normalized & 1.000 & 1.000 \\
\hline Cluster shade & $0.655^{\star}$ & 0.990 \\
\hline Cluster prominence & 0.930 & 0.851 \\
\hline Cluster tendency & 0.971 & 0.930 \\
\hline Auto correlation & 0.999 & 0.999 \\
\hline Dissimilarity & 0.996 & 0.992 \\
\hline Homogeneity1 & 0.999 & 0.999 \\
\hline Homogeneity2 & 0.990 & 0.982 \\
\hline Inverse difference normalized & 0.992 & 0.987 \\
\hline Inverse variance & 0.999 & 1.000 \\
\hline Max probability & 0.999 & 0.999 \\
\hline Variance & 0.972 & 0.932 \\
\hline Grey level nonuniformity & 1.000 & 1.000 \\
\hline Run length nonuniformity & 1.000 & 1.000 \\
\hline Low grey level run emphasis & 1.000 & 1.000 \\
\hline High grey level run emphasis & 0.999 & 0.999 \\
\hline Short run low grey level emphasis & 1.000 & 1.000 \\
\hline Short run high grey level emphasis & 0.999 & 0.999 \\
\hline Long run low grey level emphasis & 1.000 & 1.000 \\
\hline Long run high grey level emphasis & 0.999 & 0.999 \\
\hline
\end{tabular}

*, ICC <0.750. Gd-EOB-DTPA, gadolinium-ethoxybenzyl diethylenetriamine pentaacetic acid; MRI, magnetic resonance imaging. 
Table S3 The differences of clinical parameters and Gd-EOB-DTPA-enhanced MRI radiomic features based on arterial phase and hepatobiliary phase between low-grade and high-grade tumors

\begin{tabular}{|c|c|c|}
\hline Clinical parameters and radiomic features & $P$ value (arterial phase) & $P$ value (hepatobiliary phase) \\
\hline AFP & $<0.001^{*}$ & $<0.001^{*}$ \\
\hline CEA & 0.850 & 0.850 \\
\hline CA19-9 & 0.448 & 0.448 \\
\hline ALT & 0.419 & 0.419 \\
\hline AST & $0.020^{*}$ & $0.020^{*}$ \\
\hline Mean & $<0.001^{*}$ & $<0.001^{*}$ \\
\hline Standard deviation & $<0.001^{*}$ & $0.001^{*}$ \\
\hline Max frequency & $0.008^{*}$ & $0.003^{*}$ \\
\hline Mode & $<0.001^{*}$ & $<0.001^{*}$ \\
\hline Minimum & $<0.001^{*}$ & $<0.001^{*}$ \\
\hline Maximum & $<0.001^{*}$ & $<0.001^{*}$ \\
\hline The 5th percentile & $<0.001^{*}$ & $<0.001^{*}$ \\
\hline The 10th percentile & $<0.001^{*}$ & $<0.001^{*}$ \\
\hline The 25th percentile & $<0.001^{*}$ & $<0.001^{*}$ \\
\hline The 50th percentile & $<0.001^{*}$ & $<0.001^{*}$ \\
\hline The 75th percentile & $<0.001^{*}$ & $<0.001^{*}$ \\
\hline The 90th percentile & $<0.001^{*}$ & $<0.001^{*}$ \\
\hline Skewness & $0.003^{*}$ & 0.234 \\
\hline Kurtosis & $0.017^{\star}$ & 0.852 \\
\hline Entropy & $<0.001^{*}$ & $0.001^{*}$ \\
\hline AUC low & / & $0.025^{\star}$ \\
\hline S-sDlowest & $0.001^{*}$ & $<0.001^{*}$ \\
\hline S-sDav distribution width & $<0.001^{*}$ & 0.117 \\
\hline Histogram width & $<0.001^{*}$ & $0.001^{*}$ \\
\hline Area & 0.256 & $0.044^{*}$ \\
\hline Volume & 0.410 & 0.058 \\
\hline Surface area & 0.280 & $0.023^{*}$ \\
\hline compactness 1 & 0.497 & 0.120 \\
\hline compactness2 & / & $0.027^{*}$ \\
\hline Volume diameter & 0.195 & $0.019^{*}$ \\
\hline Spherical disproportion & 0.162 & 0.415 \\
\hline Sphericity & / & / \\
\hline Surface volume ratio & 0.542 & 0.210 \\
\hline Elongation & 0.233 & $0.040^{*}$ \\
\hline Entropy $(\mathrm{H})$ & $<0.001^{*}$ & $<0.001^{*}$ \\
\hline Energy & $<0.001^{*}$ & $<0.001^{*}$ \\
\hline Correlation & $<0.001^{\star}$ & $<0.001^{*}$ \\
\hline Inverse difference moment normalized & $<0.001^{*}$ & $<0.001^{*}$ \\
\hline Cluster shade & 0.076 & $0.010^{*}$ \\
\hline Cluster prominence & $<0.001^{*}$ & $<0.001^{*}$ \\
\hline Cluster tendency & $<0.001^{*}$ & $<0.001^{*}$ \\
\hline Auto correlation & $<0.001^{*}$ & $<0.001^{*}$ \\
\hline Dissimilarity & $<0.001^{\star}$ & $<0.001^{*}$ \\
\hline Homogeneity1 & $<0.001^{\star}$ & $<0.001^{*}$ \\
\hline Homogeneity2 & $<0.001^{*}$ & $<0.001^{*}$ \\
\hline Inverse difference normalized & $0.003^{*}$ & $0.001^{*}$ \\
\hline Inverse variance & $<0.001^{*}$ & $<0.001^{*}$ \\
\hline Max probability & $<0.001^{*}$ & $<0.001^{*}$ \\
\hline Variance & $<0.001^{*}$ & $<0.001^{*}$ \\
\hline Grey level nonuniformity & $0.005^{\star}$ & $0.002^{*}$ \\
\hline Run length nonuniformity & 0.446 & 0.068 \\
\hline Low grey level run emphasis & $<0.001^{*}$ & $<0.001^{*}$ \\
\hline High grey level run emphasis & $<0.001^{*}$ & $<0.001^{*}$ \\
\hline Short run low grey level emphasis & $<0.001^{*}$ & $<0.001^{*}$ \\
\hline Short run high grey level emphasis & $<0.001^{\star}$ & $<0.001^{*}$ \\
\hline Long run low grey level emphasis & $<0.001^{*}$ & $<0.001^{*}$ \\
\hline Long run high grey level emphasis & $<0.001^{*}$ & $<0.001^{*}$ \\
\hline
\end{tabular}

I, intraclass correlation coefficients $<0.750 ;{ }^{*}, \mathrm{P}<0.05$. Gd-EOB-DTPA, gadolinium-ethoxybenzyl diethylenetriamine pentaacetic acid; MRI, magnetic resonance imaging; AFP, alpha fetoprotein; CEA, carcinoembryonic antigen; CA19-9, carbohydrate antigen 19-9; ALT, alanine aminotransferase; AST, aspartate transaminase. 
A

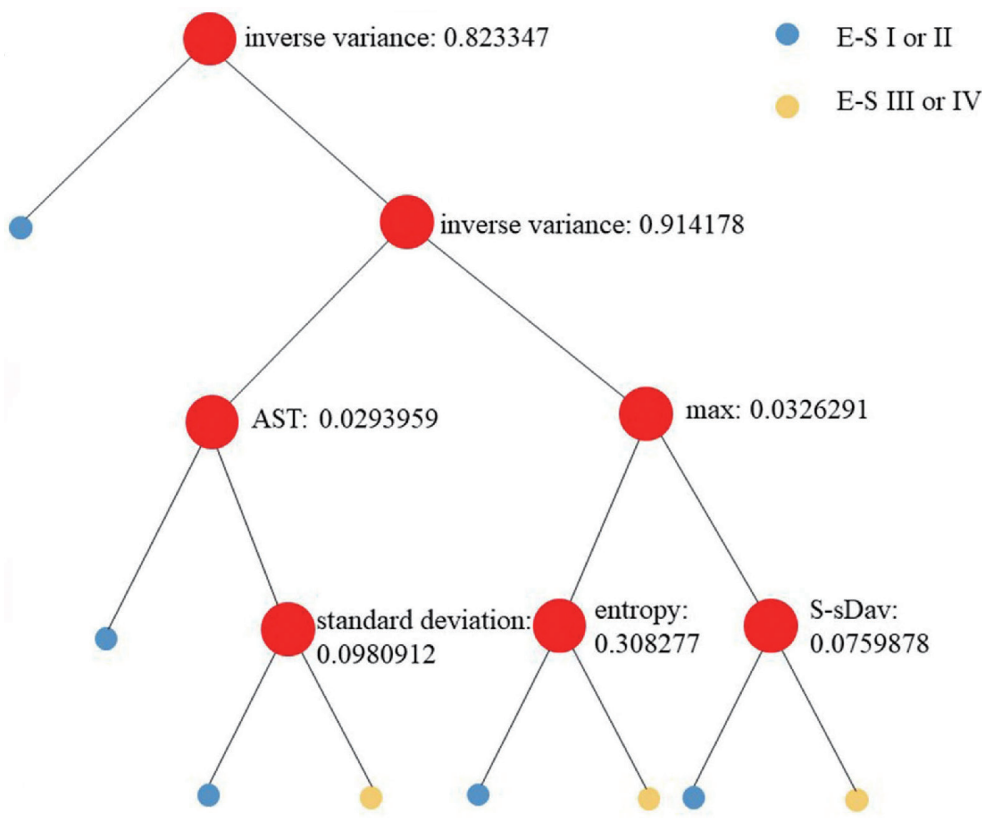

B

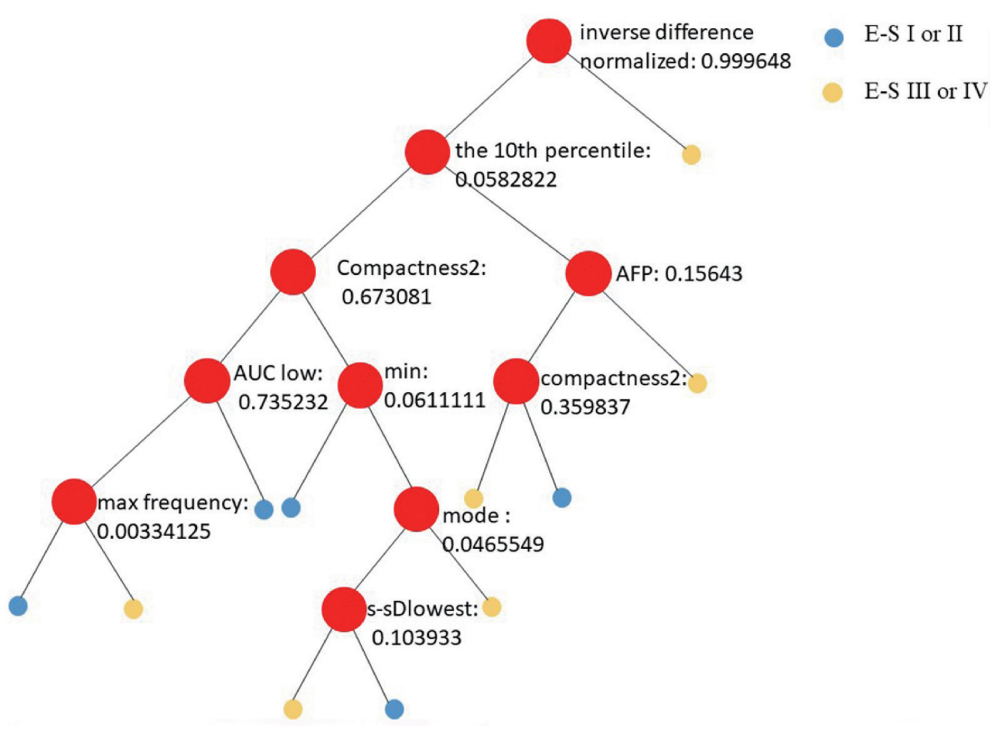

C

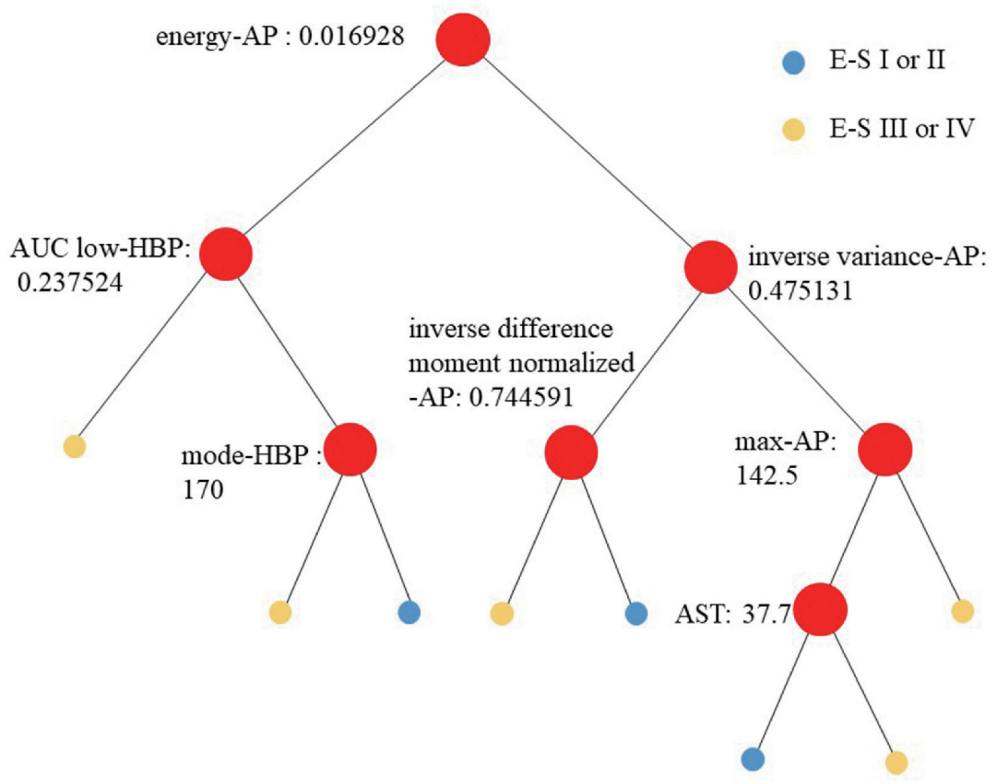

Figure S1 Decision tree of selecting clinical parameters and MRI radiomic features for the arterial phase (A), hepatobiliary phase (B), and combined arterial and hepatobiliary phase prediction models (C). 


\section{Supplement I}

Standard deviation:

standard_deviation $=\left\{\frac{1}{N-1} \sum_{i=1}^{N}[X(\mathrm{i})-\bar{X}]\right\}^{2}$

Maximum:

The maximum gray value

maximum $=\max (\mathrm{X})$

Entropy:

entropy $=\sum_{i=1}^{N_{l}} p(i) \log _{2} p(i)$

S-sDav distribution width:

Histogram width at one-half peak of the gray value histogram

Inverse variance:

inverse_variance $=\sum_{i=1}^{N_{g}} \sum_{j=1}^{N_{g}} \frac{P(i, j)}{|i-j|^{2}}, i \neq j$

Max frequency:

The maximum frequency of gray values

Mode:

Gray value of the highest peak of gray histogram

Minimum:

The minimum gray value

minimum $=\min (X)$
The 10th percentile:

The gray value is at $10 \%$, when the gray value is arranged from small to large.

\section{S-sDlowest:}

The gray value of the first frequency digit 5 that appears along the positive direction of the $\mathrm{x}$-axis

AUC low:

After removing the highest and lowest $1 \%$ gray value, the proportion of gray value lower than $25 \%$ gray value

Compactness2:

companyctness $2=36 \pi \frac{V_{2}}{A^{3}}$

Inverse difference normalized (IDN):

$I D N=\sum_{i=1}^{N_{g}} \sum_{j=1}^{N_{g}} \frac{P(i, j)}{1+\left(\frac{|i-j|}{N}\right)}$

Energy:

energy $=\sum_{i=1}^{N_{g}} \sum_{j=1}^{N_{g}}[P(i, j)]^{2}$

Inverse Difference Moment Normalized (IDMN)

$I D M N=\sum_{i=1}^{N_{g}} \sum_{j=1}^{N_{g}} \frac{P(i, j)}{1+\left(\frac{|i-j|^{2}}{N^{2}}\right)}$ 